\title{
Editorial
}

\section{The Importance of Food Perception in Food Choices and Nutrition}

Nowadays, there is a growing interest in understanding and modulating consumer choices, both for healthy and economic reasons. However, food preferences are influenced by a diversity of factors, which interact among them to a final behaviour. The understanding of food choices is linked with the comprehension of how consumers perceive food and how the different levels of perception (sensorial, psychological, socio-economic) affect those choices. Traditionally, the effect of each discipline was studied independently. Nowadays, the complexity inherent to the decision process is recognized and multidisciplinary studies, where the different dimensions of choices are considered, are increasing.

Motivation for consumption is greatly affected by the way individuals identify food. This recognition should be considered at different levels: sensorial, emotional, social and physiological. As such, variability in food characteristics, including food constituents and technological processing, as well as variations in individuals' characteristics, such as believes, experiences and physiology, and variations in the context in which food is experienced, can greatly affect the final perception.

The present thematic issue compiles together work from different disciplinary fields, which have in common, the focus on consumer food perception. Four articles were selected, among which 2 are research articles and 2 are reviews. The study by Xu provide insights into the understanding that children have about food items, nutrition and their effects on health. It is interesting to note that, although considering food and vegetables as healthy food items, they also consider some processed foods as such. These results are helpful for educational programs aimed at improving children's food perception [1]. Annunziata and Angela studied the perception consumers have about sustainability attributes of organic and local food, from where they concluded that a major portion of consumers attaches more value to attributes related to quality and health than to sustainability, with a small segment of consumers being oriented towards sustainability. Once more, educational programmes are warranted, which can benefit from this information [2].

The perception of food risk has been also focused in this thematic issue. Through an extensive review, Gaspar and da Silva Costa concluded that, food risk communication should have both promotion and prevention focus, rather than considering each of them alone [3].

Finally, the article by Rodrigues et al. focusses on another type of food perception: authenticity food perception. By reviewing research works related with the particular example of Évora cheese, the authors were able to provide evidence that, for traditional/regional food products, the existence of a Protected Designation of Origin (PDO) is responsible for different perception of consumers [4].

Overall, studies that form the presented Thematic Issue, provide evidence on the different aspects of food recognition, highlighting the need to look for consumers perception with a multidisciplinary perspective.

\section{REFERENCES}

[1] Tingting X. Young children talking about food and health. Recent Pat Food Nutr Agric 2018; 9(2): 79-86.

[2] Annunziata A, Angela M. Consumer perception of sustainability attributes in organic and local food. Recent Pat Food Nutr Agric 2018; 9(2): 87-96.

[3] Adriano da SC, Rui G. Two sizes may fit all: Promoting healthier choices through regulatory fit in risk communications. Recent Pat Food Nutr Agric 2018; 9(2): 97-103.

[4] Lénia R, Maria M, Cristina P. Cheese: Food perception and food choice. Recent Pat Food Nutr Agric 2018; 9(2): 104-10.

Dr. Elsa Lamy

(Guest Editor)

Instituto de Ciências Agrarias e Ambientais Mediterranicas (ICAAM)

Universidade de Évora

7002-554 Évora

Portugal
Dr. Fernando Capela-Silva

(Co-Guest Editor)

Instituto de Ciências Agrarias e Ambientais Mediterranicas (ICAAM) Departamento de Biologia, Escola de Ciências e Tecnologia Universidade de Evora 7000-671 Evora 